\title{
ARTICLE OPEN Zero-energy pinning from interactions in Majorana nanowires
}

\author{
Fernando Domínguez ${ }^{1}$, Jorge Cayao ${ }^{3}$, Pablo San-Jose ${ }^{3}$, Ramón Aguado ${ }^{3}$, Alfredo Levy Yeyati ${ }^{1}$ and Elsa Prada ${ }^{2}$
}

Majorana zero modes at the boundaries of topological superconductors are charge-neutral, an equal superposition of electrons and holes. This ideal situation is, however, hard to achieve in physical implementations, such as proximitized semiconducting nanowires of realistic length. In such systems Majorana overlaps are unavoidable and lead to their hybridization into charged Bogoliubov quasiparticles of finite energy, which, unlike true zero modes, are affected by electronic interactions. We here demonstrate that these interactions, particularly with bound charges in the dielectric surroundings, drastically change the non-interacting paradigm. Remarkably, interactions may completely suppress Majorana hybridization around parity crossings, where the total charge in the nanowire changes. This effect, dubbed zero-energy pinning, stabilizes Majoranas back to zero energy and charge, and leads to electronically incompressible parameter regions wherein Majoranas remain insensitive to local perturbations, despite their overlap.

npj Quantum Materials (2017)2:13; doi:10.1038/s41535-017-0012-0

\section{INTRODUCTION}

Since the early experimental efforts toward the generation and characterization of Majorana zero modes (MZMs) in nanowires, ${ }^{1-5}$ remarkable progress has been accomplished. ${ }^{6-8}$ Cleaner devices, with longer mean free paths and much more robust-induced superconductivity, are now available. Samples of this quality are expected to develop an unambiguous, topologically non-trivial superconducting phase hosting MZMs. ${ }^{9}$ Owing to their nonAbelian statistics, braiding operations of MZMs are topologically protected and hold promise as the basis of fault-tolerant quantum computers. $^{10,11}$

Majoranas are topologically protected against local fluctuations in the environment inasmuch as they do not overlap spatially. However, deviations from this stringent condition always occur in realistic samples of finite length $L$, see Fig. 1a. In this case, the two MZMs at opposite ends of the wire overlap and become a charged Bogoliubov quasiparticle of finite energy $\varepsilon_{M}$ and charge $Q_{M}<e$ (assuming a macroscopic and/or grounded parent superconductor; the case of a floating superconductor with charging energy has been studied in, e.g., refs 12,13$)$. While these deviations are expected to be exponentially small, their importance of course depends on the spatial extension of the Majoranas $\xi_{M}$ as compared to $L$ (or the wire's mean free path, whichever is smaller), since both $\varepsilon_{M}$ and $Q_{M}$ decrease as $\sim e^{-L / \xi_{M}}$.

Even in the longer $L \sim 1 \mu \mathrm{m}$ nanowires experimentally studied so far, deviations from true MZM behavior are not negligible, since typically $\varepsilon_{M}$ is in the hundreds of nanometers. This theoretical expectation is in stark contrast with many experiments reporting surprisingly robust zero-bias anomalies (see, e.g., ref. 8). Within a non-interacting picture, ${ }^{14,15}$ the only solution to this conflict is to assume values of the spin-orbit coupling much larger than the ones estimated for InAs or InSb NWs. ${ }^{16}$ Apart from the exponential suppression, $\varepsilon_{M}$ is expected to cross zero energy (parity crossings where $Q_{M}$ changes) in an oscillatory fashion ${ }^{17-19}$ as a function of magnetic field, chemical potential, or length. Both the exponential suppression and the oscillatory behavior of $\varepsilon_{M}$ have recently been demonstrated experimentally. ${ }^{7}$ However, these experiments report on unexplained features in rather short wires with $L \sim \xi_{M}$ in the form of parity crossings that extend across a finite range of magnetic field (instead of point-like zero-energy crossings).

In this work, we present an alternative to the non-interacting view that provides an explanation to the above unresolved issues. By considering electronic interactions with the electrostatic environment, ${ }^{20}$ we demonstrate that zero-energy crossings originating from the oscillatory splitting of overlapping MZMs are spontaneously stabilized into extended regions in parameter space wherein Majoranas become pinned to zero energy (Fig. 1c). Our results show that the absence of Majorana splittings, which is commonly identified with non-overlapping Majoranas with topological protection, can occur despite the Majorana overlap in nanowires of finite length.

The interactions involved in Majorana zero-energy pinning are not intrinsic to the wire, ${ }^{18,21-28}$ but rather extrinsic, between electrons that enter the wire and bound charges in the dielectric environment that arise in response. Our results suggest that such electronic interactions provide a powerful mechanism to stabilize Majorana-based qubits in realistic nanowires, and may account for the hitherto unexplained experimental features. ${ }^{7,8}$

\section{RESULTS}

The central idea behind the zero-energy pinning phenomenon is the emergence of repulsive self-interactions through image charges in the dielectric medium. Before presenting full microscopic calculations, we first illustrate the mechanism with a toy example. Assume a quantum system with a single-particle state $\psi_{M}$ that carries an electric charge $Q_{M}$. In the absence of interactions its energy is $\varepsilon_{M}$. The Hamiltonian, including a "self-interaction" term,

\footnotetext{
'Departamento de Física Teórica de la Materia Condensada, Condensed Matter Physics Center (IFIMAC) and Instituto Nicolás Cabrera, Universidad Autónoma de Madrid, Madrid E-28049, Spain; ${ }^{2}$ Departamento de Física de la Materia Condensada, Condensed Matter Physics Center (IFIMAC) and Instituto Nicolás Cabrera, Universidad Autónoma de Madrid, Madrid E-28049, Spain and ${ }^{3}$ Instituto de Ciencia de Materiales de Madrid, Consejo Superior de Investigaciones Científicas (ICMM-CSIC), Sor Juana Inés de la Cruz 3, Madrid 28049, Spain

Correspondence: Elsa Prada (elsa.prada@uam.es)
}

Received: 13 September 2016 Revised: 5 January 2017 Accepted: 22 January 2017

Published online: 07 March 2017 
(a)
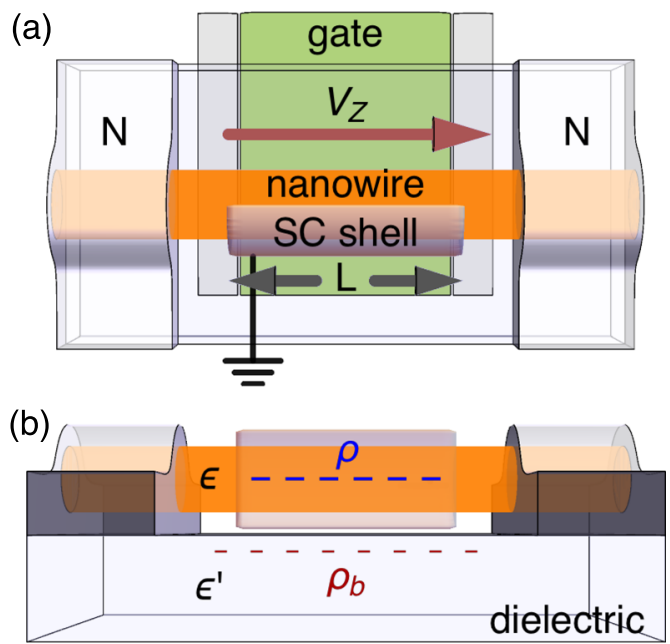



Fig. 1 a Proximitized Rashba nanowire of length $L$ with gate-tunable Fermi energy $\mu$ and under a parallel Zeeman field $V_{z}$. b Bound charges $\rho_{b}$ arise in the dielectric surroundings, which interact with free charges $\rho$ in the nanowire. c Sketch of the interaction-induced pinning, discussed in this work, of Majoranas at zero energy around parity crossings. This pinning provides a natural explanation to hitherto unresolved experimental features in Majorana wires (see, e. g., Fig. 3c in ref. 7)

takes the form $H=\varepsilon_{M} \psi_{M}^{\dagger} \psi_{M}+V_{b} Q_{M}\left\langle\psi_{M}^{\dagger} \psi_{M}\right\rangle \psi_{M}^{\dagger} \psi_{M}$, with $e V_{b}$ an energy scale. Thus, in the presence of interactions, the effective level $\tilde{\varepsilon}_{M}$ is the self-consistent solution to $\tilde{\varepsilon}_{M}=\varepsilon_{M}+V_{b} Q_{M} f\left(\tilde{\varepsilon}_{M}\right)$, where $f(\varepsilon)=1 /\left(e^{\varepsilon / k_{B} T}+1\right)$ is the Fermi function. As $\varepsilon_{M}$ is externally tuned by a parameter $V_{Z}$ to cross the Fermi energy (defined as zero), the resulting electron and hole energies $\pm \tilde{\varepsilon}_{M}$ with interactions (solid curves, $V_{b}>0$ [repulsive]) and without (dashed, $V_{b}=0$ ) are of the form shown in Fig. 1c for $T \rightarrow 0$. We see that the $V_{b}=0$ parity crossing at $\varepsilon_{M}\left(V_{Z}\right)=0$ transforms, for repulsive $V_{b}>0$, into a finite plateau (in red), wherein $\tilde{\varepsilon}_{M}$ becomes pinned to zero within a finite range of $V_{Z}, 0<\varepsilon_{M}\left(V_{Z}\right)<V_{b} Q_{M}$. The pinning plateau is electronically incompressible, and is the result of thermal equilibrium combined with the self-interaction energy cost of occupying state $\psi_{M}$.

The above toy model for pinning presents a fundamental question when considering Majoranas in the role of the $\psi_{M}$ state: how do physical self-interactions arise? It is clear that selfinteractions of the form $\left\langle\psi_{M}^{\dagger} \psi_{M}\right\rangle \psi_{M}^{\dagger} \psi_{M}$ are unphysical in an isolated quantum system. In a generic basis, a direct (intrinsic) charge-charge interaction of the form $H_{C}=\sum_{i j} \psi_{i}^{\dagger} \psi_{i} V_{i j} \psi_{j}^{\dagger} \psi_{j}$ produces self-interaction of eigenstates when treated at the Hartree level, but this is canceled by the Fock correction. ${ }^{29-31}$

However, if the electronic system is immersed in a dielectric medium, a bound charge density $\rho_{b}(\boldsymbol{r})$ may appear, in response to electric charges $\hat{\rho}(r)$ in the system, at interfaces where the dielectric constant $\varepsilon(\boldsymbol{r})$ changes, see Fig. $1 \mathrm{~b}$. These $\rho_{b}(\boldsymbol{r})$ generate an electrostatic potential $\phi(\boldsymbol{r})$ that acts back onto $\hat{\rho}(\mathbf{r})$, such that the total potential $\phi_{\text {tot }}(\boldsymbol{r})=\phi_{\text {sys }}(\boldsymbol{r})+\phi(\boldsymbol{r})$ satisfies the inhomogeneous Poisson equation $\boldsymbol{\nabla} \cdot\left[\varepsilon(r) \nabla \phi_{\text {tot }}(r)\right]=-4 \pi\langle\hat{\rho}(r)\rangle$, and $\phi_{\text {sys }}(r)$ is the potential for an infinite system with a uniform $\varepsilon$ (without bound charges). The resulting (extrinsic) interaction between the system's $\hat{\rho}(\mathbf{r})$ and bound charges $\rho_{b}\left(\boldsymbol{r}^{\prime}\right)$ then takes the form of a Hartee-like physical self-interaction, $\int \phi(r) \hat{\rho}(r)=\int V_{b}\left(r^{\prime}, r\right)\left\langle\hat{\rho}\left(r^{\prime}\right)\right\rangle \hat{\rho}(r)$, where $V_{b}$ depends on the actual device geometry. Note that no Fock-like correction should be included here, since this is a purely classical effect: bound charges are located outside the nanowire and can be distinguished from the free charges. Thus, an effective Hamiltonian similar to the toy model above becomes relevant. Interaction with bound charges should be expected to produce zero-energy pinning of a quantum state as long as they are repulsive (i.e., if $\rho_{b}$ and $\rho$ have the same sign). The latter condition is satisfied if the dielectric environment has a smaller dielectric constant than the nanowire, the typical situation in most experiments (e.g., InSb or InAs nanowires on a $\mathrm{SiO}_{2}$ substrate, see the "Discussion" section for further details). Such selfinteractions are well known in the context of molecular junctions, ${ }^{32,} 33$ but, to our knowledge, their implications have not been explored in the context of Majorana wires.

A second relevant question concerns the charge $Q_{M}$ associated to Majoranas. MZMs in infinite superconducting systems are charge-neutral, an equal superposition of electrons and holes. As such, it might come as a surprise that electronic interactions, which are sensitive to electronic charge, would have an effect on Majoranas. It was demonstrated, ${ }^{34,} 35$ however, that the state resulting from the hybridization of two overlapping MZMs, each located at an end of a nanowire of length $L$, is not charge-neutral, but rather defines a charged Bogoliubov quasiparticle of finite energy $\varepsilon_{M}$ and charge $Q_{M}<e$, with typical $\varepsilon_{M}$ and $Q_{M}$ decreasing exponentially as $\sim e^{-L / \xi_{M}}$. We now analyze this phenomenology in detail within a non-interacting model ${ }^{14,15}$ of a proximitized InSb semiconducting nanowire, oriented along the $x$-direction, of length $L=1 \mu \mathrm{m}$ and subjected to a parallel Zeeman field $V_{Z}=$ $g \mu_{B} B / 2$ ( $g$ is the $g$-factor, $\mu_{B}$ is the Bohr magneton, and $B$ is the magnetic field). The continuum Hamiltonian without superconductivity reads $H_{0}=\hbar^{2} k^{2} / 2 m+a \sigma_{y} k+V_{z} \sigma_{x}$, where $\hbar k$ is the momentum along the wire, $\boldsymbol{\sigma}$ is the spin, the InSb spin-orbit coupling is $a=0.2 \mathrm{eV} \AA$, and the effective mass is $m=0.015 m_{e}$. We include the induced superconducting pairing $\Delta=0.5 \mathrm{meV}$ in the second-quantized Nambu representation,

$H=\frac{1}{2} \int d x\left(\Psi^{\dagger}(x), \Psi(x)\right)\left(\begin{array}{cc}H_{0}-\mu-i \sigma_{y} \Delta^{*} \\ i \sigma_{y} \Delta & \mu-H_{0}^{*}\end{array}\right)\left(\begin{array}{c}\Psi(x) \\ \Psi^{\dagger}(x)\end{array}\right)$,

where $\Psi(x)=\left(\Psi_{\uparrow}(x), \Psi_{\downarrow}(x)\right)$ is the electron field, so that the charge density reads $\hat{\rho}(x)=e \sum_{\sigma} \Psi_{\sigma}^{\dagger}(x) \Psi_{\sigma}(x)$.

Figure $2 \mathrm{a}$ and $\mathrm{b}$ show the total charge in the nanowire $Q_{\text {tot }}=$ $\int_{0}^{L} d x\langle\hat{\rho}(x)\rangle$ at zero temperature and the low-energy spectrum as a function of $V_{Z}$, respectively. Any given Bogoliubov quasiparticle eigenstate

$\psi_{n}=\int_{0}^{L} d x\left[u_{n}(x) \Psi(x)+v_{n}(x) \Psi^{\dagger}(x)\right]$

in the Nambu spectrum with energy $\varepsilon_{n}>0$ contributes to $Q_{\text {tot }}=\Sigma_{n} Q_{n}$ with a charge

$Q_{n}=e \int_{0}^{L} d x\left\{f\left(\varepsilon_{n}\right)\left|u_{n}(x)\right|^{2}+\left[1-f\left(\varepsilon_{n}\right)\right]\left|v_{n}(x)\right|^{2}\right\}$, 

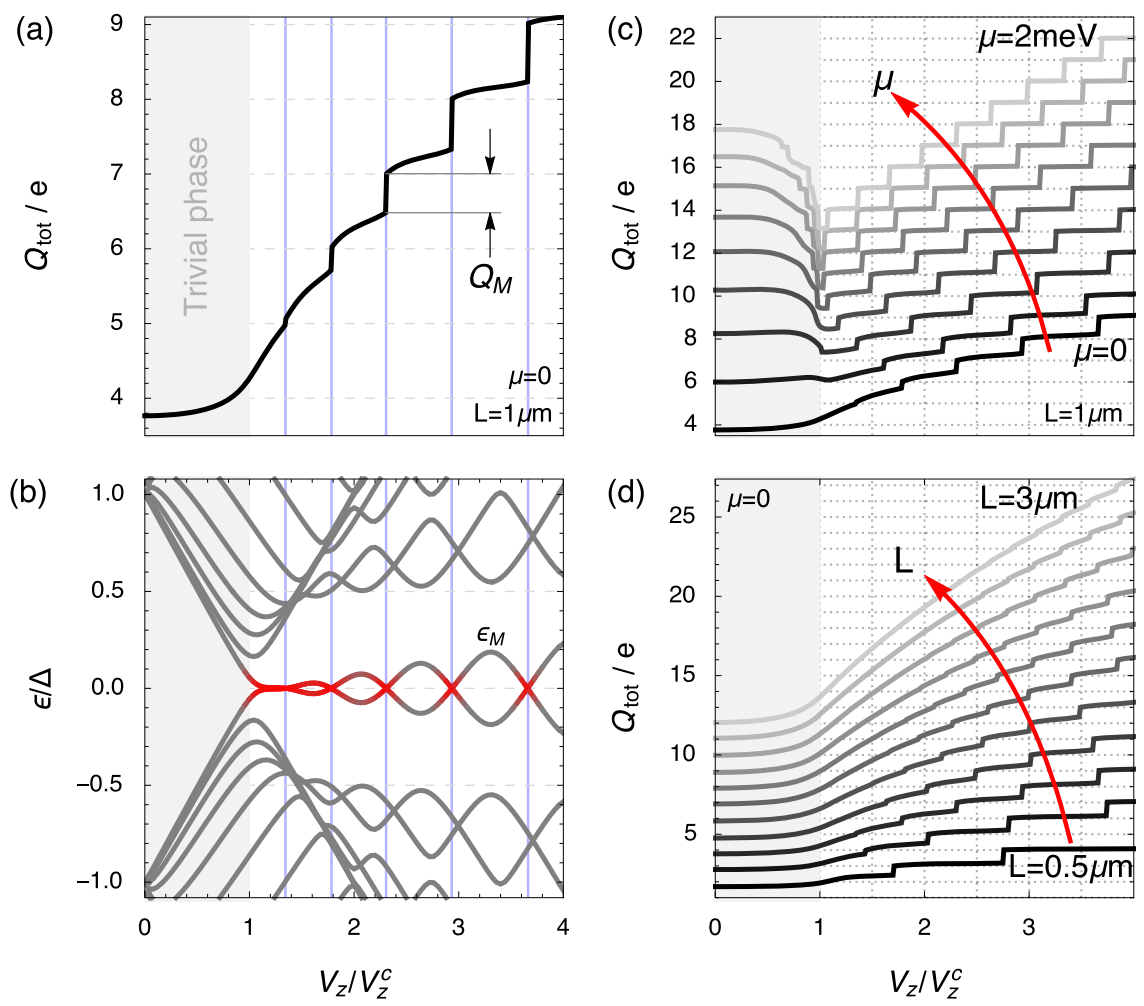

Fig. 2 a Total charge $Q_{\text {tot }}$ in a non-interacting topological nanowire vs. $V_{Z}$. For $V_{Z}>V_{Z}{ }^{c}, Q_{\text {tot }}$ increases in jumps of magnitude $Q_{M}<e$ at parity crossings, shown in red in b. c, d Charge jumps become more (less) quantized as $\mu(L)$ increases

where $f\left(\varepsilon_{n}\right)$ is its occupation probability. For $V_{Z}>V_{Z}^{c}=\sqrt{\Delta^{2}+\mu^{2}}$ the nanowire becomes topologically non-trivial and, for $L \rightarrow \infty$, develops MZMs at each end $\gamma_{L, R}=\gamma_{L, R}^{\dagger}$ with $u_{L, R}(x)=v_{L, R}^{*}(x)$. These states have zero charge, since changing their occupation does not change $Q_{\text {tot }}$. For finite $L$, the MZMs overlap and are no longer eigenstates, but hybridize into two special $n= \pm 1 \mathrm{Nambu}$ eigenstates $\psi_{1}=\psi_{M}=\left(\gamma_{L}+i \gamma_{R}\right) / 2$ and $\psi_{-1}=\psi_{M}^{\dagger}=\left(\gamma_{L}-i \gamma_{R}\right) / 2$, whose energies $\pm \varepsilon_{M}$ oscillate around zero as a function of $V_{Z}, \mu$, or $L$. Each time $\varepsilon_{M}$ crosses zero in Fig. $2 b$ (parity crossing), the zerotemperature occupation of the $\psi_{M}$ quasiparticle at equilibrium changes abruptly, and the total charge of the wire experiences a non-quantized jump $Q_{M}=\left|Q_{1}-Q_{-1}\right|$, as shown in Fig. $2 a$. Thus, the charge $Q_{M}$ of the non-local Majorana fermion $\psi_{M}$ is non-zero despite it being a superposition of the two neutral MZMs, and is distributed almost uniformly along the wire. It reads

$$
\begin{array}{r}
Q_{M}=\frac{e}{4} \int_{0}^{L} d x\left[\left|u_{L}^{*}(x)+i u_{R}^{*}(x)\right|^{2}-\left|u_{L}^{*}(x)-i u_{R}^{*}(x)\right|^{2}\right]= \\
e \int_{0}^{L} d x\left|u_{L}(x) u_{R}(x)\right|,
\end{array}
$$

i.e., $Q_{M}$ is the spatial overlap of the two Majoranas (here we have chosen $u_{L}$ real and $u_{R}$ imaginary without loss of generality). As we have discussed in the "Introduction", the spatial extension of MZMs $\xi_{M}$ is typically larger than the spin-orbit length $L_{S O}=\hbar^{2} / a m^{36}$ which is seldom smaller than a few hundred nanometers $\left(L_{\mathrm{sO}}=\right.$ $254 \mathrm{~nm}$ here). In the weak SO regime, which corresponds to typical SO lengths for InSb nanowires of $L_{S O} \approx 250 \mathrm{~nm}$ (SO coupling $a \sim 0.2 \mathrm{eVA})$, the Majorana localization length is $\xi_{M} \sim L_{S O}$, and furthermore increases with Zeeman field. ${ }^{37}$ The condition $L \gg \xi_{M}$ cannot be fulfilled as the Zeeman field exceeds the critical value above the topological transition. If, on the other hand, we assume stronger SO couplings, $\xi_{M}$ saturates to $\xi_{M} \sim a / \Delta,{ }^{16,36}$ which, for typical values of $\Delta$, is still much larger than the SO length. For example, assuming a SO coupling ten times larger than before, $a \sim 2 \mathrm{eVA}$, and a proximity gap $\Delta=0.5 \mathrm{meV}$ (of the order of the experimental one in ref. 8), the Majorana localization length is $\xi_{M} \sim 400 \mathrm{~nm}$. The $L \gg \xi_{M}$ limit would therefore need much longer wires than the ones studied so far. Thus, even in rather long $L \sim 1$ $\mu \mathrm{m}$ non-interacting topological nanowires, the overlap of the Majoranas is sizeable, and results in large $Q_{M} \sim e$ charge jumps (see Fig. 2a).

Since parity-crossings introduce a charge $Q_{M}$ into the system, they should be expected to give rise, in a repulsive dielectric environment, to bound charges of the same sign and hence to repulsive self-interactions and to zero-energy pinning of the corresponding MZMs. To demonstrate this effect we now add interactions to the above InSb Rashba nanowire model. Following the preceding discussion, we include only interactions with bound charges in the dielectric medium, described at a self-consistent Poisson mean field level. (The addition of intrinsic interactions do not change the essential results, and are analyzed in the Supplementary Information.) We replace $H_{0}$ in the non-interacting nanowire Hamiltonian $H$ above with $H_{0}+\phi(x)$, where the selfconsistent potential created by bound charges is written as

$\phi(x)=\int d x^{\prime} V_{b}\left(x, x^{\prime}\right)\left\langle\hat{\rho}\left(x^{\prime}\right)\right\rangle$.

We assume a simple geometry and a Coulomb interaction with image charges of the form

$$
V_{b}\left(x, x^{\prime}\right) \approx\left(\varepsilon-\varepsilon^{\prime}\right) /\left(\varepsilon+\varepsilon^{\prime}\right) /\left(4 \pi \varepsilon_{0} \varepsilon\left((2 R)^{2}+\left|x-x^{\prime}\right|^{2}\right)^{1 / 2}\right),
$$

where $\varepsilon=17.7$ is the $\operatorname{lnSb}$ dielectric constant, $\varepsilon^{\prime}=3.9$ is that of a $\mathrm{SiO}_{2}$ substrate, and $R=50 \mathrm{~nm}$ is the nanowire radius (see Supplementary Information for a derivation). The results are qualitatively independent of the precise form of $V_{b}$, as long as it is repulsive $\left(\varepsilon>\varepsilon^{\prime}\right)$. We reabsorb the $V_{Z}=0$ value of the potential $\phi(L / 2)$ at the center of the nanowire into $\mu$, so that the actual Fermi energy at zero magnetic field is equal to $\mu$ with and without interactions.

The potential $\phi(x)$ is solved numerically by self-consistent iteration (see the Supplementary Information for analytical results). 

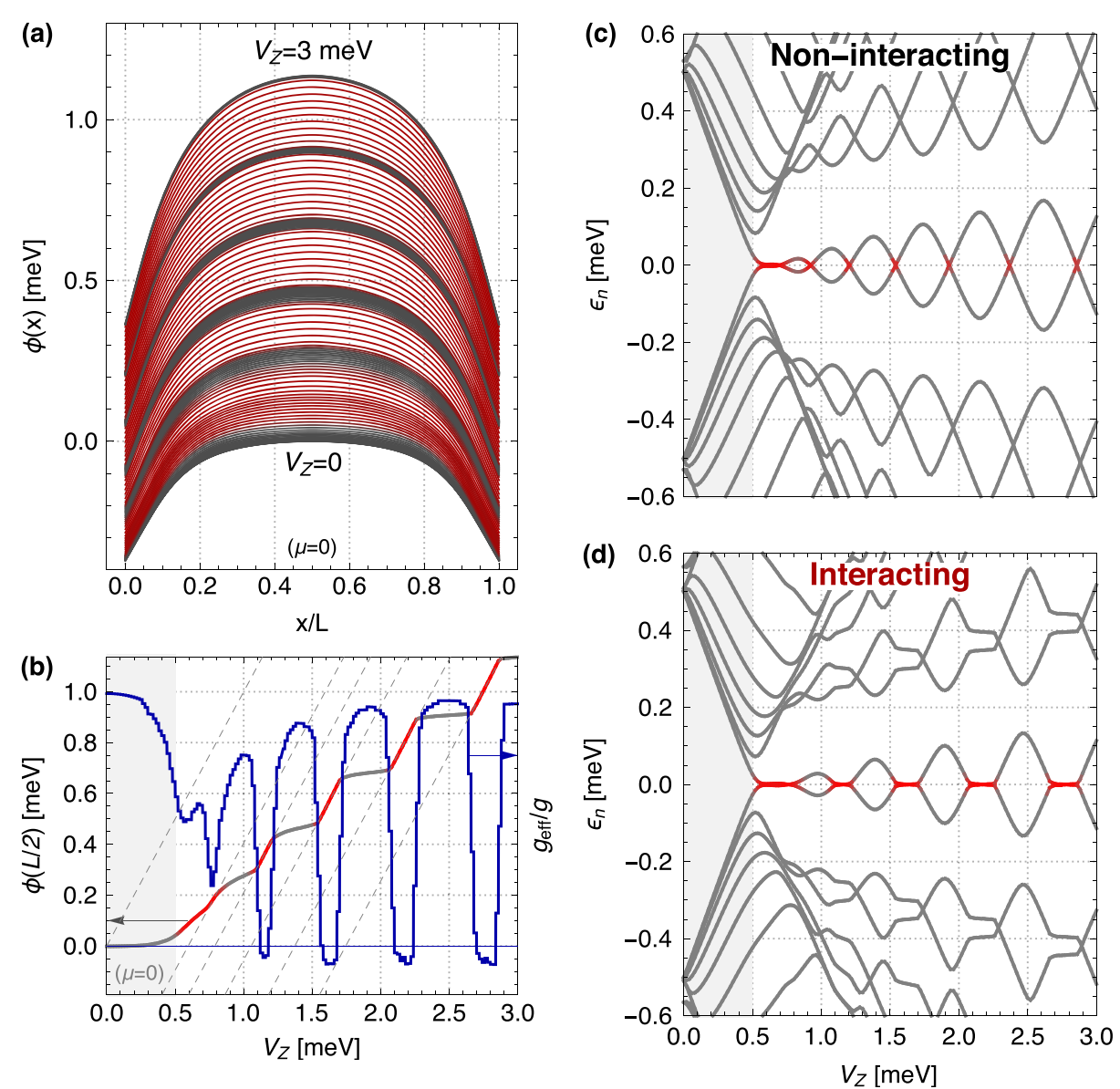

Fig. 3 a Screening potential $\phi(x)$ along a proximitized $\operatorname{lnSb}$ nanowire of radius $R=50 \mathrm{~nm}$ and length $L=1 \mu \mathrm{m}$ for increasing Zeeman field $V_{Z}$. We take $\Delta=0.5 \mathrm{meV}$ and $\mu=0$. Red (gray) curves correspond to an incompresible (compressible) regime. $\mathbf{b}$ Increase of the potential at the center of the wire $\phi(L / 2)$ with $V_{Z}$ and the corresponding effective g-factor $g_{\text {eff }}=g\left[1-\partial \phi(L / 2) / \partial V_{z}\right]$ (blue line). Intervals in red correspond to almost perfect screening of $V_{Z}\left(g_{\text {eff }} \approx 0\right)$. c, $\mathbf{d}$ Non-interacting and interacting spectra of the nanowire. Note that perfect Zeeman screening by interactions is correlated with zero-energy pinnings of Majorana bound states (extended zero modes in red). An equivalent simulation to d including also intrinsic interactions in the nanowire is shown in Supplementary Material Fig. 1

The solution for $\mu=0$ is shown in Fig. $3 a$ as a function of $V_{z}$, while $\phi$ $(L / 2)$ evaluated at the center of the nanowire is shown in Fig. $3 \mathrm{~b}$. The $V_{Z}$-dependent $\phi(L / 2)$ transforms the simple non-interacting Fermi energy $\varepsilon_{F}=\mu+V_{Z}$ into a non-trivial $\varepsilon_{F}^{\text {eff }}=\mu+V_{Z}-\phi(L / 2)$ at the center of the nanowire, changing also the $V_{Z}$-dependence of the spectrum as a result. Panels (c) and (d) show the corresponding low-energy spectrum without and with interactions, respectively. For $V_{z}>V_{z}{ }^{c}=0.5 \mathrm{meV}$, parity crossings emerge in the noninteracting spectrum that inject a finite charge $Q_{M}$ into the system (Fig. 2a). At each of these points, the interactions with bound charges conspire to suppress the charging, giving rise to finite intervals of $V_{Z}$ with pinned zero-energy modes in place of parity crossings (panel (d)). These are the result of a rapid increase in the overall self-consistent $\phi(x)$ within each interval (red curves in panels $(a, b)$ ), which screens the Zeeman field in $\varepsilon_{F}^{\text {eff }}$ almost completely, making the system incompressible $\partial \varepsilon_{F}^{\text {eff }} / \partial \mathrm{V}_{Z} \approx 0$. This intermittent incompressibility is visible in Fig. $3 \mathrm{~b}$ as a slope $\partial \phi(L / 2) / \partial V_{Z} \approx 1$. The electronic compressibility against variations of $V_{z}$ may be quantified by the effective g-factor $g_{\text {eff }}=g \partial \varepsilon_{F}^{\text {eff }} / \partial V_{Z}=g\left[1-\partial \phi(L / 2) / \partial V_{Z}\right]$, in blue. Deviations from exact zero-energy pinning and perfect incompressibility $g_{\text {eff }}=0$ may arise from finite temperature, $T=10 \mathrm{mK}$ in these simulations, or finite decay rate into the reservoirs, neglected here.

In the above simulation the chemical potential was taken as $\mu=0$. A similar phenomenology persist also at different electronic densities. Figure 4 shows the incompressible pinned regions (panels $(\mathrm{a}, \mathrm{b})$, in red) and total charge $Q_{\text {tot }}$ in the nanowire (panels (c, d)) across the full $\mu-V_{z}$ parameter space, without and with interactions. When interactions are switched on, zero-measure parity crossings grow into extended areas of pinned MZMs, always separated by areas with finite MZM hybridization $\left|\tilde{\varepsilon}_{M}\right|>0$ (white). Along a given line within each incompressible area, the charge in the nanowire jumps by a finite, almost constant $Q_{M}$.

We note that, apart from stabilizing MZMs, interactions induce a change around $\mu=0$ in the slope $s$ of incompressible regions in the $\mu-V_{z}$ plane, with $s=-1$ for $\mu<0$ like in the non-interacting case, and $-1<s<0$ for $\mu>0$. The incompressible regions are contours of constant $\varepsilon_{F}^{\text {eff }},{ }^{36}$ so that $s=-g \kappa_{\text {eff }} / g_{\text {eff }}$ is a ratio between system's $\mu$-compressibility $\kappa_{\text {eff }}=\partial \varepsilon_{F}^{\text {eff }} / \partial \mu$, and the $V_{Z}$-compressibility $g_{\text {eff }} / g=\partial \varepsilon_{F}^{\text {eff }} / \partial V_{Z}$ discussed above. While both are equal for $\mu<0, \kappa_{\text {eff }}$ becomes suppressed for $\mu>0$, so that $s \approx-1 /\left(1+(v / \mu)^{1 / 2}\right)$ for a constant $v \propto V_{b}{ }^{2}$ related to the interaction strength (see Supplementary Information).

\section{DISCUSSION}

We have discussed a generic mechanism whereby electrostatic interactions with the dielectric surroundings stabilize zero-energy modes in a finite length Majorana nanowire. This zero-energy pinning effect is the result, within a self-consistent mean field, of a repulsive self-interaction of nanowire electrons that arises when its dielectric environment has a smaller dielectric constant than the nanowire itself. In a more general situation in which the charge screening by the normal contacts and the parent 

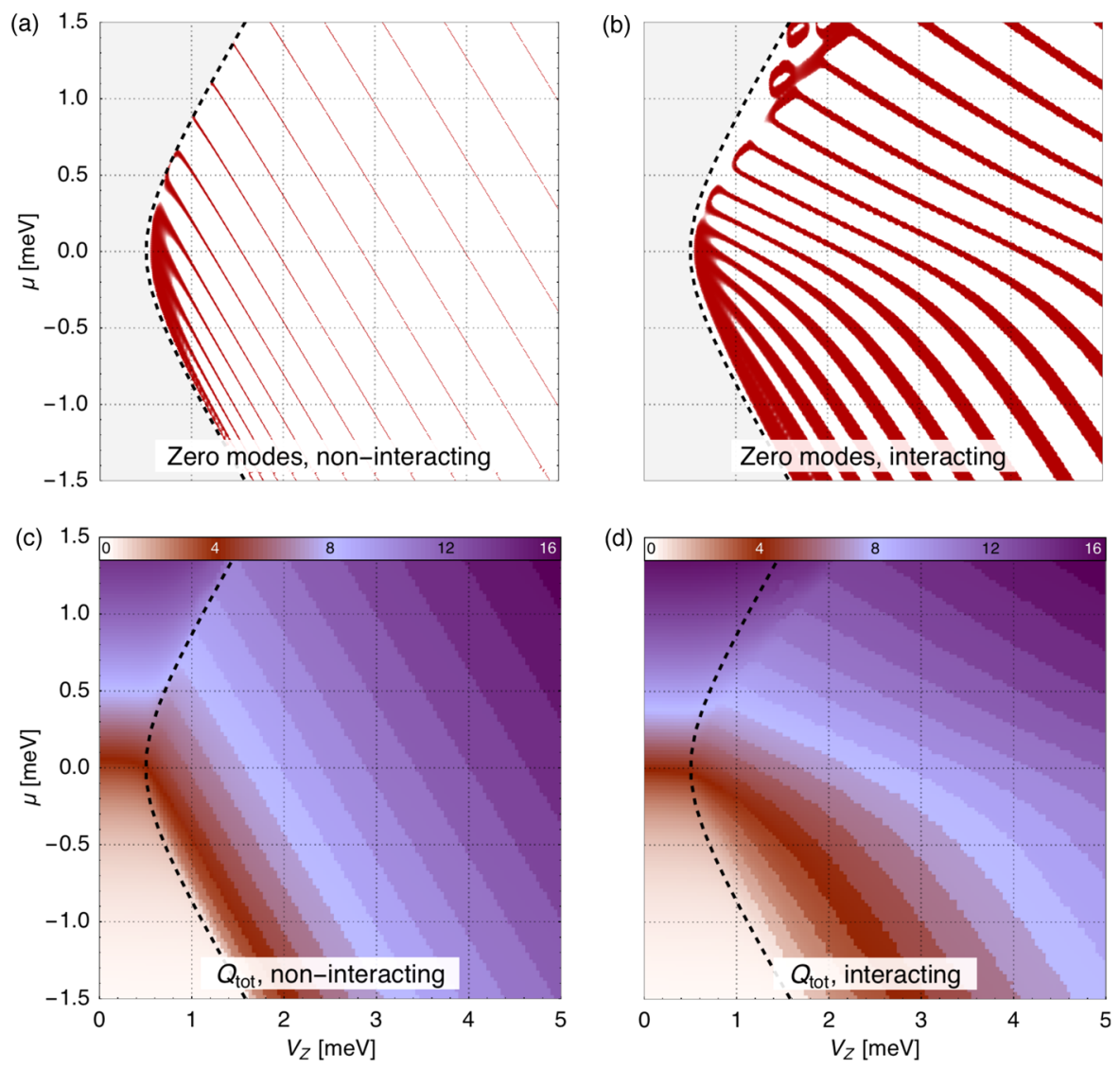

Fig. 4 Topological phase diagram of a non-interacting $(\mathbf{a}, \mathbf{c})$ and interacting $(\mathbf{b}, \mathbf{d})$, finite-length nanowire, see Fig. 3 for parameters. The dashed line corresponds to the non-interacting topological transition $V_{z}^{c}=\left(\Delta^{2}+\mu^{2}\right)^{1 / 2}$. Red areas in $\mathbf{a}, \mathbf{b}$ represent zero-energy modes (with energy below $10 \mu \mathrm{eV}$ at $T=10 \mathrm{mK}$ ), which correspond to narrow parity crossings from hybridized MZMs in a, and extended incompressible regions of MZMs pinned at zero energy in $\mathbf{b}$. The total charge in the nanowire, panels $\mathbf{c}$, $\mathbf{d}$, increases by $Q_{M}$ jumps at each zero-energy crossing

superconductor is also considered (a situation not presented here for simplicity, but with qualitatively similar results), the relevant quantity becomes the difference between the total electrostatic energy of a charge inside and outside the proximitized nanowire. This energy difference becomes positive, and hence selfinteractions are repulsive, if charge screening is reduced upon entering the proximitized region. Such is the natural situation for nanowires with partial superconducting shells but full metallic covering at the contacts. The addition of charge into the nanowire calculated self-consistently then leads to pinning. Related dielectric-induced changes in addition energies have been discussed in molecular single electron transistors. ${ }^{32}$ Zero-energy pinning cannot be captured by solving the electrostatic problem for infinite nanowires, ${ }^{20}$ since it is necessary to take into account the electrostatic energy cost of adding charge at each parity crossing. Pinning, moreover, does not require a single channel regime. It also operates for any odd number of open channels, ${ }^{38-40}$ as the physics of parity crossings is similar.

Within the stabilized regions in parameter space with pinned Majoranas, the system becomes electronically incompressible (a related phenomenon of global incompressibility, albeit unconnected to zero-energy pinning and parity crossings, was discussed in Majorana nanowires in the limit of strong intrinsic interactions $\left.{ }^{18}\right)$. As a result, potential fluctuations $\delta \mu$ from the environment or fluctuations $\delta V_{Z}$ in the applied Zeeman field become screened out by interactions. This should remain true even for spatially non-uniform perturbations. As a result, the pinning effect could potentially be exploited to protect realistic Majorana-based qubits against environmental noise. We anticipate that, by carefully engineering the dielectric surroundings of finite-length Majorana nanowires, one could exploit the electronic incompressibility of pinned regions to replace, at least partially, the topological protection of MZMs against decoherence, which is lost by their overlap. In this respect it is important to emphasize that pinned MZMs still overlap spatially, but become locked into degenerate parity eigenstates, regardless of any local perturbation, as long as thermal equilibrium is preserved. We should stress that, despite the common misconception in large part of the literature that identifies robust zero Majorana splitting with topological protection, it is non-locality what ultimately renders fermionic parity qubits immune to local noise. While there is a strong resemblance, the resilience to arbitrary perturbations discussed in this work is different from topological protection. The implications of pinning for the decoherence and relaxation times of parity-conserving Majorana qubits, such as, e.g., the $|00\rangle$ and $|11\rangle$ even-parity states of a four-Majorana setup, are nontrivial and should be the subject of future work.

The zero-energy pinning mechanism described in this work is generic, and is the result of the electrostatic energy cost from the interaction of the finite charge $Q_{M}$ added to the nanowire at parity crossings and image charges in the dielectric environment. Given the generality of the mechanism, we speculate that the ideas discussed here are also relevant in other contexts, including parity crossings of Shiba states in non-topological superconductors.

\section{METHODS}

All the numerical results were computed within a self-consistent meanfield treatment of interactions in a tight-binding model for the 
semiconducting nanowire. We used the MathQ framework ${ }^{41}$ on a spatial discretization of the nanowire model $H_{0}=\hbar^{2} k^{2} / 2 m+a \sigma_{y} k+V_{z} \sigma_{x}$. The lattice spacing used is $10 \mathrm{~nm}$. The mean field self-consistency condition, encoded in Eq. (4), is achieved by iteration with an adaptive update coefficient. This is required at low temperatures to achieve convergence around pinned regions.

\section{ACKNOWLEDGEMENTS}

We thank K. Flensberg for illuminating discussions. We acknowledge financial support from the Spanish Ministry of Economy and Competitiveness through the Ramón y Cajal program RYC-2013-14645 and RYC-2011-09345, grant Nos. FIS201233521, FIS2013-47328-C2-1-P, FIS2014-55486-P, FIS2015-65706-P, FIS2015-64654-P (MINECO/FEDER), and the "María de Maeztu" Program for Units of Excellence in R\&D (MDM-2014-0377)

\section{AUTHOR CONTRIBUTIONS}

F.D., J.C., P.S-J., and E.P. performed the calculations. P.S-J. developed the MathQ computational framework. All authors contributed to the discussion, the design of the research, the analysis of the results, and the writing of the manuscript.

\section{COMPETING INTERESTS}

The authors declare no competing interest.

\section{REFERENCES}

1. Mourik, V. et al. Signatures of Majorana fermions in hybrid superconductorsemiconductor nanowire devices. Science 336, 1003-1007 (2012).

2. Deng, M. T. et al. Anomalous zero-bias conductance peak in a Nb-InSb nanowire-Nb hybrid device. Nano. Lett. 12, 6414-6419 (2012).

3. Das, A. et al. Zero-bias peaks and splitting in an al-inas nanowire topological superconductor as a signature of Majorana fermions. Nat. Phys. 8, 887-895 (2012).

4. Churchill, H. O. H. et al. Superconductor-nanowire devices from tunneling to the multichannel regime: zero-bias oscillations and magnetoconductance crossover. Phys. Rev. B 87, 241401 (2013).

5. Lee, E. J. H. et al. Spin-resolved andreev levels and parity crossings in hybrid superconductor-semiconductor nanostructures. Nat. Nano. 9, 79-84 (2014).

6. Chang, W. et al. Hard gap in epitaxial semiconductor-superconductor nanowires. Nat. Nano. 10, 232-236 (2015)

7. Albrecht, S. M. et al. Exponential protection of zero modes in Majorana islands. Nature 531, 206-209 (2016).

8. Zhang, $\mathrm{H}$. et al. Ballistic Majorana nanowire devices. arXiv:1603.04069v1 (2016).

9. Kitaev, A. Y. Unpaired Majorana fermions in quantum wires. Phys. Usp 44, 131 (2001)

10. Nayak, C., Simon, S., Stern, A., Freedman, M. \& Das Sarma, S. Non-Abelian anyons and topological quantum computation. Rev. Mod. Phys. 80, 1083-1159 (2008).

11. Sarma, S. D., Freedman, M. \& Nayak, C. Majorana zero modes and topological quantum computation. NPJ Quantum Inf. 1, 15001 EP (2015).

12. $\mathrm{Fu}, \mathrm{L}$. Electron teleportation via Majorana bound states in a mesoscopic superconductor. Phys. Rev. Lett. 104, 056402 (2010).

13. Hützen, R., Zazunov, A., Braunecker, B., Yeyati, A. L. \& Egger, R. Majorana singlecharge transistor. Phys. Rev. Lett. 109, 166403 (2012).

14. Lutchyn, R. M., Sau, J. D. \& Das Sarma, S. Majorana fermions and a topological phase transition in semiconductor-superconductor heterostructures. Phys. Rev. Lett. 105, 077001 (2010).

15. Oreg, Y., Refael, G. \& von Oppen, F. Helical liquids and Majorana bound states in quantum wires. Phys. Rev. Lett. 105, 177002 (2010).

16. Mishmash, R. V., Aasen, D., Higginbotham, A. P. \& Alicea, J. Approaching a topological phase transition in Majorana nanowires. Phys. Rev. B 93, 245404 (2016).

17. Prada, E., San-Jose, P. \& Aguado, R. Transport spectroscopy of ns nanowire junctions with Majorana fermions. Phys. Rev. B 86, 180503(R) (2012).

18. Das Sarma, S., Sau, J. D. \& Stanescu, T. D. Splitting of the zero-bias conductance peak as smoking gun evidence for the existence of the Majorana mode in a superconductor-semiconductor nanowire. Phys. Rev. B 86, 220506 (2012).
19. Rainis, D., Trifunovic, L., Klinovaja, J. \& Loss, D. Towards a realistic transport modeling in a superconducting nanowire with Majorana fermions. Phys. Rev. B 87, 024515 (2013)

20. Vuik, A., Eeltink, D., Akhmerov, A. R. \& Wimmer, M. Effects of the electrostatic environment on the Majorana nanowire devices. New J. Phys. 18, 033013 (2016).

21. Stoudenmire, E. M., Alicea, J., Starykh, O. A. \& Fisher, M. P. Interaction effects in topological superconducting wires supporting Majorana fermions. Phys. Rev. B 84, 014503 (2011).

22. Lutchyn, R. M. \& Fisher, M. P. A. Interacting topological phases in multiband nanowires. Phys. Rev. B 84, 214528 (2011).

23. Sela, E., Altland, A. \& Rosch, A. Majorana fermions in strongly interacting helical liquids. Phys. Rev. B 84, 085114 (2011).

24. Gangadharaiah, S., Braunecker, B., Simon, P. \& Loss, D. Majorana edge states in interacting one-dimensional systems. Phys. Rev. Lett. 107, 036801 (2011).

25. Fidkowski, L., Alicea, J., Lindner, N. H., Lutchyn, R. M. \& Fisher, M. P. A. Universal transport signatures of Majorana fermions in superconductor-luttinger liquid junctions. Phys. Rev. B 85, 245121 (2012).

26. Manolescu, A., Marinescu, D. C. \& Stanescu, T. D. Coulomb interaction effects on the Majorana states in quantum wires. J. Phys.: Condens. Matter 26, 172203 (2014).

27. Ghazaryan, A. \& Chakraborty, T. Long-range coulomb interaction and Majorana fermions. Phys. Rev. B 92, 115138 (2015).

28. Xu, H., Xiong, Y. \& Wang, J. Topological phases and transport properties of screened interacting quantum wires. Phys. Lett. A 380, 3534-3540 (2016).

29. Cowan, R. D. Atomic self-consistent-field calculations using statistical approximations for exchange and correlation. Phys. Rev. 163, 54-61 (1967).

30. Perdew, J. P. \& Zunger, A. Self-interaction correction to density-functional approximations for many-electron systems. Phys. Rev. B 23, 5048-5079 (1981).

31. Anisimov, V. I. Strong Coulomb Correlations in Electronic Structure Calculations (CRC Press, 2000).

32. Kaasbjerg, K. \& Flensberg, K. Strong polarization-induced reduction of addition energies in single-molecule nanojunctions. Nano. Lett. 8, 3809-3814 (2008).

33. Kaasbjerg, K. \& Flensberg, K. Image charge effects in single-molecule junctions: breaking of symmetries and negative-differential resistance in a benzene singleelectron transistor. Phys. Rev. B 84, 115457 (2011).

34. Ben-Shach, G. et al. Detecting Majorana modes in one-dimensional wires by charge sensing. Phys. Rev. B 91, 045403 (2015).

35. Lin, C. H., Sau, J. D. \& Das Sarma, S. Zero-bias conductance peak in Majorana wires made of semiconductor/superconductor hybrid structures. Phys. Rev. B 86, 224511 (2012).

36. Klinovaja, J. \& Loss, D. Composite Majorana fermion wave functions in nanowires. Phys. Rev. B 86, 085408 (2012).

37. Cayao, J., Prada, E., San-Jose, P. \& Aguado, R. SNS junctions in nanowires with spin-orbit coupling: role of confinement and helicity on the subgap spectrum. Phys. Rev. B 91, 024514 (2015).

38. Potter, A. C. \& Lee, P. A. Engineering a p+ip superconductor: comparison of topological insulator and rashba spin-orbit-coupled materials. Phys. Rev. B 83, 184520 (2011)

39. Stanescu, T. D., Lutchyn, R. M. \& Das Sarma, S. Majorana fermions in semiconductor nanowires. Phys. Rev. B 84, 144522 (2011).

40. San-Jose, P., Prada, E. \& Aguado, R. Mapping the topological phase diagram of multiband semiconductors with supercurrents. Phys. Rev. Lett. 112, 137001 (2014).

41. San-Jose, P. MathQ, a Mathematica package for the simulation of quantum systems, www.icmm.csic.es/sanjose/MathQ/MathQ.html.

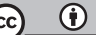

This work is licensed under a Creative Commons Attribution 4.0 International License. The images or other third party material in this article are included in the article's Creative Commons license, unless indicated otherwise in the credit line; if the material is not included under the Creative Commons license, users will need to obtain permission from the license holder to reproduce the material. To view a copy of this license, visit http://creativecommons.org/licenses/by/ $4.0 /$

(c) The Author(s) 2017

Supplementary Information accompanies the paper on the npj Quantum Materials website (doi:10.1038/s41535-017-0012-0). 